\title{
25 Research Soure \\ Identification of Key Genes and Biological Pathways of Tumor-infiltrating CD8+ T Cell in Lung Adenocarcinoma
}

\section{Lingling Bao}

Zhejiang University School of Medicine First Affiliated Hospital Beilun Branch

\section{Kai Sun}

Liuzhou people's hospital

Xuede Zhang ( $\sim$ zxd1978cn@163.com)

Zhejiang University School of Medicine First Affiliated Hospital Beilun Branch https://orcid.org/00000002-0112-4529

\section{Research article}

Keywords: lung adenocarcinoma, microRNAs, tumor-derived exosomes, tumor immune response, regulatory network

Posted Date: February 21st, 2022

DOI: https://doi.org/10.21203/rs.3.rs-961645/v1

License: (9) (1) This work is licensed under a Creative Commons Attribution 4.0 International License. Read Full License 


\section{Abstract}

Background: The immune microenvironment of lung cancer is complex, and T-cell tolerance is a primary mechanism of immune evasion. Tumor-derived exosomes (TEX) miRNAs regulate immune cells in tumor microenvironment (TME), contributing to tumor immune suppression. However, the underlying mechanisms remain unclear.

Methods: TEX miRNA expression dataset (GSE111803) and CD8+ T cells mRNA expression profile (GSE90728) in lung adenocarcinoma (LUAD) were downloaded from Gene Expression Omnibus (GEO). The differentially expressed miRNAs (DEMs) and differentially expressed genes (DEGs) were analyzed. The intersection of target genes of DEMs and DEGs was defined as the overlapped target genes. We performed the function and pathway analysis for DEGs and the overlapped genes by the DAVID tool and constructed a protein-protein interaction (PPI) network to screen modules and hub genes.

Results: In total, 7 DEMs (5 upregulated and two downregulated miRNAs) and 672 DEGs (219 upregulated and 453 downregulated genes) were identified, then 185 overlapped genes for upregulated DEMs were selected for further analysis. GO analysis indicated the DEGs were mainly enriched in positive regulation of $B$ cell activation, innate immune, while the overlapped genes were primarily involved in regulation of immune response. The pathway analysis suggested DEGs were primarily enriched in cytokine-cytokine receptor interaction, while the overlapped genes were primarily enriched in osteoclast differentiation. The PPI network revealed three significant modules for DEGs and the overlapped genes sets. These modules genes were mainly enriched in cell cycle, chemokine signaling pathway, and cytokine-cytokine receptor interaction, respectively.

Conclusions: These results preliminarily revealed the mRNA expression profiles and the molecular regulatory mechanism of TEX miRNA regulated tumor immune response in LUAD.

\section{Background}

Lung cancer is one of the most common malignancies with the highest mortality globally, and lung adenocarcinoma (LUAD) is the most common pathological subtype of non-small-cell lung cancer (NSCLC)[1]. The tumor microenvironment plays a vital role in cancer progression and outcome. Exosomes, a key mediator of intercellular communication, regulated various malignant phenotypes, including inducing an immunosuppressive microenvironment and promoting tumor immune escape[2-4]. Tumor-derived exosomes (TEX) may carry immunosuppressive molecules, such as proteins DNA, mRNA, and miRNAs, to immune cells, thereby interfering with immune cell functions to promote tumor progression[5]. Recent studies indicated that miRNAs are abundant in exosomes, suggesting that miRNAs might play potential roles in mediating intercellular communication and immunoregulation in the tumor microenvironment. [6, 7].

In the past few years, many studies have suggested exosomal miRNAs can affect mRNA expression/translation in immune cells and form a basis for immune suppression[8]. Tumor-derived 
exosomal miR-23a, as an immunosuppressive factor, targeted the expression of CD107a in NK cells and reduced anti-tumor immune response[9]. A recent study has indicated that exosomal miR-214 downregulated PTEN expression in T cells, promoting regulatory T cell (Treg) differentiation and enhancing Treg-mediated immunity in murine lung cancer and sarcoma models[10]. Therefore, it is worthwhile to construct a tumor-derived exosomal miRNA-target gene regulatory network to investigate underlying mechanisms of immune regulation in the tumor microenvironment (TME). Moreover, CD8+ cells were the main lymphocyte populations in TME. Hence, it is valuable to explore the mechanisms of tumor-derived exosomal miRNA regulating tumor-infiltrating CD8+ T cell in TME, which facilitates finding the new strategies for LUAD immunotherapy.

In the present study, the miRNA expression profile in the peripheral exosomes of LUAD (GSE111803) and purified populations of CD8+ T cells mRNA expression profile (GSE90728) in NSCLC were downloaded from Gene Expression Omnibus (GEO, http://www.ncbi.nlm.nih.gov/geo/). The differentially expressed miRNA (DEMs) were identified, and target genes of DEMs were predicted. Subsequently, the differentially expressed genes (DEGs) were screened, and the overlapped genes between target genes of DEMs and DEGs were defined. Finally, GO and KEGG analysis and miRNA-target gene regulatory network were performed to investigate the potential mechanisms of tumor-derived exosomal miRNA regulating CD8+ $T$ cell in TME of LUAD.

\section{Materials And Methods}

Ethical approval or patient consent was unnecessary since the present study was an integrative analysis of published data.

\section{Microarray data}

The miRNA expression profile of GSE111803 and the gene expression profile (GSE90728) were obtained from the GEO database (http://www.ncbi.nlm.nih.gov/geo/). The GSE111803 dataset was based on GPL16791 Illumina HiSeq 2500, including five serum exosome samples of lung adenocarcinoma and five healthy controls.[11] The GSE90728 based on GPL16791 Illumina HiSeq 2500 contained 36 NSCLC tissues and 32 adjacent lung tissue, and 27 LUAD tissues and 24 adjacent lung tissue were included in the present study.

\section{Identification of DEMs and DEGs}

BioJupies is a web application for analyzing RNA-seq data, allowing users to generate customized, interactive analysis results through an intuitive user interface[12]. In the study, the datasets of GSE111803 and GSE90728 were analyzed by the BioJupies website. DEMs and DEGs were defined according to $\mathrm{P}<0.05$ and $|\mathrm{Log} 2 \mathrm{FC}| \geq 1.5$.

\section{Target genes prediction of DEMs}

Two databases, miRPathDB 2.0 [13] and TargetScan, were used to predict target genes of DEMs.[14].In this study. Genes identified in these two databases were considered as predicted target genes of DEMs. In 
order to acquire more specific target genes, the intersection of predicted target genes of DEMs and DEGs was considered as overlapped target genes.

\section{GO and KEGG analysis}

The DEGs and overlapped target genes were used for GO and Kyoto Encyclopedia of Genes and Genomes (KEGG) enrichment analysis using the Database for Annotation, Visualization, and Integrated Discovery (DAVID) online tool[15]. $P<0.05$ was considered statistically significant.

\section{Protein-protein interaction (PPI) network and modules analysis}

The Search Tool for the Retrieval of Interacting Genes (STRING; http://string.embl.de/) database was used to construct functional interaction networks of DEGs and overlapped target genes with the interaction score $>0.7[16]$. The PPI network was visualized using cytoscape, and the plug-in cytoHubba was applied to identify hub genes[17]. According to the degree of genes in the PPI network, the top 20 ranked genes were considered as hub genes. The Molecular Complex Detection (MCODE) plug-in was applied to screen modules in the PPI network. The criteria were set as follows: degree cutoff $=2$, node score cutoff $=0.2, \mathrm{k}$-core $=2$ and max depth $=100$. In order to investigate the potential molecular mechanism of exosomal miRNAs regulating CD8+ in TME of LUAD, we construct a miRNA-target genes regulatory network using DEMs and overlapped target genes through cytoscape software.

\section{Results}

\section{Screening for DEMs and DEGs}

In this study, 7 DEMs ( 5 upregulated miRNAs and 2 downregulated miRNAs) were identified from GSE111803 in blood exosome of LAUD compared to healthy controls. The DEMs were listed in Table 1. The volcano plot of DEMs was shown in Figure 1A. In total, 672 DEGs (219 upregulated genes and 453 downregulated genes) were screened in CD8+ T cells of LUAD samples compared with adjacent lung tissue. (All DEGs are listed in Supplementary Material 1). The volcano plot of DEMs was shown in Figure 1B. 
Table 1

DEMs in serum exosome of LAUD compared to healthy controls

\begin{tabular}{|c|c|c|c|c|}
\hline miRNA_ID & log2FoldChange & AveExpr & $t$ & P.Value \\
\hline \multicolumn{5}{|c|}{ Upregulated miRNAs } \\
\hline hsa-miR-1180-3p & 1.930964 & 4.862005 & 3.355627 & 0.005201 \\
\hline hsa-miR-4732-5p & 1.899286 & 3.615943 & 2.878671 & 0.012988 \\
\hline hsa-miR-486-3p & 1.77035 & 13.42185 & 3.757349 & 0.002416 \\
\hline hsa-miR-486-5p & 1.694508 & 13.53942 & 3.768195 & 0.002366 \\
\hline hsa-miR-1255b-5p & 1.504928 & 3.636998 & 2.774006 & 0.015864 \\
\hline \multicolumn{5}{|c|}{ Downregulated miRNAs } \\
\hline hsa-miR-369-3p & -1.53774 & 5.18293 & -2.37412 & 0.033772 \\
\hline hsa-miR-410-3p & -1.50819 & 3.833375 & -2.9767 & 0.010764 \\
\hline
\end{tabular}

\section{Prediction of DEMs target genes}

To elucidate DEMs regulatory mechanism in CD8+T cells in TME of LUAD, the target genes were predicted for upregulated DEMs using two databases (miRPathDB 2.0 and TargetScan), and the intersection of these two gene sets was considered as miRNA target genes. Subsequently, 12467 target genes and 8720 target genes for upregulated DEMs were predicted by miRPathDB 2.0 and TargetScan, respectively. Finally, the intersection of the DEGs and target genes for DEMs was performed by Venn analysis and were considered as overlapped target genes (Figure 2). Venn diagram analysis showed 185 overlapped target genes for upregulated DEMs for further study.

\section{GO and KEGG analysis}

GO and KEGG analysis was performed on the DEGs and overlapped target genes. The top ten $G 0$ and KEGG terms of DEGs were shown in Figure 3. In biological process (BP) group, the upregulated DEGs were mainly enriched in positive regulation of $B$ cell activation, phagocytosis, recognition, and $B$ cell receptor signaling pathway (Figure $3 \mathrm{~A}$ ). While the downregulated DEGs were mainly enriched in inflammatory response, innate immune response, and cell adhesion (Figure 3E). In cellular components (CC) group, the upregulated DEGs were primarily involved in blood microparticle, immunoglobulin complex, circulating, and external side of plasma membrane (Figure 3B). The downregulated DEGs were mainly enriched in plasma membrane, integral component of plasma membrane, and extracellular space (Figure 3F). In the molecular function (MF) group, the upregulated DEGs were primarily enriched in immunoglobulin receptor binding, antigen binding, and serine-type endopeptidase activity (Figure 3C). The downregulated DEGs were mainly enriched in IgG binding, receptor activity, and carbohydrate binding (Figure 3G). KEGG pathway enrichment analysis revealed the upregulated DEGs were significantly 
enriched in cell cycle, cytokine-cytokine receptor interaction and ECM-receptor interaction (Figure 3D), while the downregulated DEGs were mainly involved in osteoclast differentiation, phagosome and Cytokine-cytokine receptor interaction (Figure H).

Subsequently, we also performed GO and KEGG analysis for overlapped target genes. The top ten GO and KEGG terms of overlapped target genes were shown in Figure 4. For BP group, the overlapped target genes were significantly enriched in respiratory gaseous exchange, regulation of immune response, and chemotaxis (Figure 4A). For CC group, the overlapped target genes were significantly enriched in plasma membrane, integral component of plasma membrane, and integral component of membrane (Figure 4B). For MF group, the overlapped target genes were significantly enriched in receptor activity, low-density lipoprotein receptor activity, and RNA polymerase II core promoter proximal region sequence-specific DNA binding (Figure 4C). KEGG pathway enrichment analysis revealed the overlapped target genes were significantly enriched in osteoclast differentiation, staphylococcus aureus infection and complement and coagulation cascades (Figure 4D)

\section{PPI network and modules analysis}

The PPI network of DEGSs and overlapped target genes was constructed by the STRING online database. Subsequently, the PPI network was visualized by cytoscape software, and hub genes were screened by degree methods through plug-in cytoHubba. The top 20 ranked genes were listed in Table 2. (Figure 5AC). Three significant modules for upregulated DEGs, downregulated DEGs, and overlapped target genes were obtained using MCODE, respectively. (Figure 5D-F). The KEGG pathway enrichment analysis of the three modules genes was performed. The for upregulated DEGs were mainly enriched in cell cycle, progesterone-mediated oocyte maturation, p53 signaling pathway, and oocyte meiosis. The module for downregulated DEGs was mainly enriched in chemokine signaling pathway, cytokine-cytokine receptor interaction, neuroactive ligand-receptor interaction, and staphylococcus aureus infection. The module for overlapped target genes was mainly enriched in chemokine signaling pathway, cytokine-cytokine receptor interaction, and neuroactive ligand-receptor interaction. (Figure 5G-I). Finally, the 5 upregulated exosomal miRNAs and overlapped target genes were selected to construct miRNA-target genes regulatory network of tumor-infiltrating CD8+ T cell in lung adenocarcinoma. (Figure 6). 
Table 2

The top 20 ranked hub genes

\begin{tabular}{|c|c|c|c|c|c|c|}
\hline \multirow[t]{2}{*}{ Gene sets } & \multicolumn{2}{|c|}{ Upregulated genes } & \multicolumn{2}{|c|}{ Downregulated genes } & \multicolumn{2}{|c|}{ Overlapped target genes } \\
\hline & Gene symbol & degree & Gene symbol & degree & Gene symbol & degree \\
\hline \multirow[t]{20}{*}{ Hub genes } & CDK1 & 38 & ITGAM & 20 & CKAP4 & 10 \\
\hline & BUB1 & 34 & PTAFR & 19 & LILRB2 & 10 \\
\hline & CCNB1 & 33 & CKAP4 & 19 & FCGR3A & 9 \\
\hline & CCNA2 & 32 & FPR1 & 18 & CSF1R & 8 \\
\hline & KIF11 & 29 & FN1 & 18 & S1PR1 & 8 \\
\hline & ASPM & 28 & FCER1G & 17 & PTAFR & 7 \\
\hline & BUB1B & 27 & S1PR1 & 16 & CX3CR1 & 7 \\
\hline & DLGAP5 & 27 & LILRB2 & 16 & OLR1 & 6 \\
\hline & $\mathrm{KIF} 2 \mathrm{C}$ & 26 & CXCR1 & 15 & CYBB & 6 \\
\hline & CENPF & 26 & PIK3R2 & 15 & VCAN & 6 \\
\hline & NCAPG & 25 & PDGFB & 15 & FGR & 6 \\
\hline & TTK & 24 & CYBB & 14 & C5AR1 & 6 \\
\hline & TOP2A & 24 & FPR3 & 14 & CXCL16 & 6 \\
\hline & RRM2 & 24 & CD33 & 14 & CXCR2 & 6 \\
\hline & CEP55 & 23 & TIMP1 & 13 & P2RY13 & 6 \\
\hline & PBK & 23 & SIRPA & 13 & HCAR2 & 6 \\
\hline & MELK & 22 & APOE & 13 & SERPINA1 & 5 \\
\hline & CENPE & 22 & OLR1 & 13 & CST3 & 5 \\
\hline & KIF4A & 22 & MCEMP1 & 13 & SIGLEC14 & 5 \\
\hline & TPX2 & 22 & SERPINA1 & 13 & SIRPA & 5 \\
\hline
\end{tabular}

\section{Discussion}

Tumor immune evasion is one of the hallmarks of cancer and can promote tumor progression. However, the potential molecular mechanism of how tumors escape the immune response remains poorly understood. Tumor cells created an immune-suppressive tumor microenvironment and promoted cell proliferation, survival, invasion, and metastasis[18]. The immunophenotypes based on CD8+ T cells in TME are helpful to understand the tumor immune escape[19]. 
The tumor microenvironment comprises immune cells, tumor cells, and the surrounding stroma[20]. Tumor growth requires remodeling immune and stromal cells, establishing a favorable tumor microenvironment. TEX might transport miRNAs into the immune and stromal cells from the tumor cells, creating an immunosuppressive microenvironment that facilitates tumor survival through escaping immune surveillance[21]. Therefore, it may help develop new therapeutic strategies and improve prognosis to explore the regulatory mechanism of tumor-derived exosomal miRNAs targeting immune cells in TME of LUAD.

To explore the gene expression profiles in CD8+ cells in TME of LUAD, we identified DEGs between LUAD tissues and normal lung tissues, then GO and KEGG pathway analysis was conducted for these DEGs. The function enrichment analysis indicated that the upregulated DEGs were primarily involved in positive regulation of $B$ cell activation, $B$ cell receptor signaling pathway, and cytokine-cytokine receptor interaction, while the downregulated DEGs were mainly enriched in inflammatory response and innate immune response. The results indicated the infiltrating CD8+ T cells are reprogramed in TME, affecting the anti-tumor immune response and tumor progression. Many studies suggested innate immune cells affected the activity of CD8+T cells by releasing factors to TME, such as TGF- $\beta$ [22], TNF- $\alpha[23]$, IL-12[24], and IFN[25], and activated adaptive immunity.

KEGG pathway analysis also revealed the upregulated DEGs were significantly enriched in cell cycle, cytokine-cytokine receptor interaction and ECM-receptor interaction, while the downregulated DEGs were mainly involved in osteoclast differentiation, phagosome, and Cytokine-cytokine receptor interaction. The cell cycle time of CD8(+) T cells is controlled by the antigenic stimulus provided by the APC and affects the rate of $T$ cell expansion and the number of effector $T$ cells[26]. Many biological processes are regulated by cytokines, including immunoregulatory activities, cell growth, differentiation, and immunosuppressive properties[27, 28]. Moreover, we also screened hub genes in upregulated DEGs, such as CDK1, CCNB1, CCNA2, BUB1B, and TOP2A. The expression levels of CDK1 and CCNB2 are correlated with the immune infiltration of CD8 T cells in hepatocellular carcinoma and indicate a worse prognosis[29]. BUB1B was reported to contribute to the resistance of CD8+ T-cell infiltration in hepatocellular carcinoma[30].

Interestingly, the pathway of phagosome was enriched in downregulated DEGs. It was reported that tumor-derived autophagosomes were efficient carriers of tumor antigens and induced efficient CD8 + T cell responses, and exert an antitumor effect[31,32]. Our results indicated downregulated DEGs play a tumor-promoting function through phagosome pathway. We also identified hub genes in downregulated DEGs, including FN1, TIMP-1, and FPR1. FN1, as tumor antigens, might contribute to enhancing T-cell response in lung cancer cells[33]. TIMP-1 might enhance the infiltrative capacity of T leukemia cell lines[34], and FPR1 induced anticancer immune responses[35]. Our findings suggested the downregulated might inhibit T cell infiltrating in TME of LUAD.

In our study, a total of 7 DEMs, including 5 upregulated miRNAs (hsa-miR-1180-3p, hsa-miR-4732-5p, hsamiR-486-3p, hsa-miR-486-5p, and hsa-miR-1255b-5p) and 2 downregulated miRNAs (hsa-miR-369-3p and 
hsa-miR-410-3p) were screened in the serum exosome of LADU. Many studies showed the expression of miR-486-5p was dysregulated in lung cancers,but these results are inconsistent. A previous study indicated miR-486-5p was under-expressed in NSCLC tissues [36-38]. However, many studies have shown miR-486-5p expression was remarkably elevated in the serum of NSCLC [39-41]. The likely reason for this is that some circulating miRNA drives from active secretion, which results in the deviation between tissue and plasma. A limited number of studies concerning the involvement of miR-486-5p in the tumor immune response have been reported. However, miR-486 can be considered predictive biomarkers for vaccine treatment efficacy in colorectal cancer[42]. It was reported that miR-1180-3p was upregulated in hepatocellular carcinoma[43], lung adenocarcinoma cells[44], and early gastric cancer[45], while the effects of miR-1180-3p might be involved in lung inflammatory signaling and immune response[46]. The miR-4732-5p and miR-1255b-5p were also found to be elevated in ovarian cancer[47] ,breast cancer[48], and bladder cancer[49], respectively. It was reported that hsa-miR-486-3p and hsa-miR-4732-5p might be involved in immune modulation by regulating gene expression in immune cells[50]. However, the relationship between these miRNAs and tumor immune was not reported.

To explore the potential mechanisms of exosomal miRNAs regulating CD8+ T cell in TME, we predicted downregulated DEMs' target genes, acquired the intersection of target genes and DEGs, then performed GO and pathway analysis. Our findings indicated the overlapped target genes were significantly enriched in osteoclast differentiation, staphylococcus aureus infection and complement and coagulation cascades pathways. The osteoclast differentiation pathway was also identified for downregulated DEGs, indicating downregulated DEMs might play an important role in regulating $C D 8+T$ cell through targeting the overlapped target genes.

Osteoclasts originate from haemopoietic stem cells and human peripheral blood-derived osteoclasts can also prime T-cells[51]. Numerous studies have highlighted bone and immune cells are regulated by partially overlapping regulatory mechanisms[52-54]. Previous studies revealed that staphylococcus aureus toxin suppressed antigen-specific $T$ cells and affected the host acquired immune response[55, 56]. The complement and coagulation cascades pathway was also significantly associated with the tumor mutational burden (TMB) scores and immune infiltration in bladder cancer[57]. The above results suggested overlapped target genes were involved in the immune response by various pathways.

Finally, we constructed a PPI network for overlapped target genes and screened a significant module, which was involved in chemokine signaling pathway, cytokine-cytokine receptor interaction, and neuroactive ligand-receptor interaction. The results revealed the DEMs regulated $C D 8+T$ cells expression in TME of LUAD by chemokine and cytokine, including CX3CR1, CXCR2, and CXCL16. It was reported that CX3CR1-CD8+ T cells played essential roles in anti-tumor efficacy but were functionally suppressed in the TME[58]. Chemokine CXCL16 can activate and attract tumor-infiltrating CD8 effector T cells and plays a vital role in exerting an anti-tumor effect in breast and colorectal cancer[59,60]. In our study, we screened the top 20 hub genes according to degree. Among these hub genes, P2RY13 was highly correlated with various tumor-infiltrating immune cells and immune functions in TME. [61-63]. P2RY13, a G proteincoupled receptor, was involved in the metabolism of extracellular ATP, which is the main source of 
adenosine in the TME[62]. The ATP-adenosine pathway functions as a key modulator of immune response within the TME, affecting the activity of various immune cells in the tumor[64]. However, the effect of ATP-adenosine pathway on tumor-infiltrating immune cells is complex, depending on the immune cell subset, purinergic receptor types, and level of ATP/adenosine[65].A previous study showed OLR1 was expressed on tumor-infiltrating immune cells and significantly associated with PD-L1, T cell dysfunction, and exclusion potential[66]. S1PR1, as a feature of tissue-resident memory T cells, plays a vital role in lymphocyte migration, affecting cytotoxicity of activated CD8(+) T-lymphocytes[67]. On the other hand, it has also been shown that S1PR1 promotes the accumulation of Tregs, inhabits CD8+ T cell infiltration and function, and affects the TME[68]. It was reported that VCAN activated tumor-intrinsic WNT pathway and enhanced CD8+ T cell infiltration in colorectal cancer[69]. Moreover, many studies indicated C5AR1[70] and CXCR2[71] were involved in tumor immunity. Until now, the influence of these DEMs on CD8+ T cells was not reported in the literature. Although our findings revealed these exosomal miRNAs might be involved in tumor immune response, the conclusion still needs to be confirmed by experimental data.

\section{Conclusions}

In summary, we screened tumor-derived exosomal DEMs, identified DEGs of CD8+ T cells in LUAD. Subsequently, we analyzed the biological functions and signaling pathways for the overlapped genes between target genes of DEMs and DEGs. In addition, we constructed a PPI network for the overlapped genes, then identified hub genes and significant modules. Our results provided a new theoretical basis for how miRNA in exosomes regulated tumor immune response in LUAD. However, more experimental evidence is required to validate the underlying regulatory mechanisms.

\section{Abbreviations}

TEX: Tumor-derived exosomes; TME: Tumor microenvironment; LUAD: Lung adenocarcinoma; GEO: Gene Expression Omnibus; DEMs: Differentially expressed miRNAs; DEGs: Differentially expressed genes; PPI: Protein-protein interaction; NSCLC: Non-small-cell lung cancer; Treg: Regulatory T cells; KEGG: Kyoto Encyclopedia of Genes and Genomes; DAVID: Database for Annotation, Visualization, and Integrated Discovery; STRING: Search Tool for the Retrieval of Interacting Genes; MCODE: Molecular Complex Detection; BP: Biological process; CC: Cellular components; MF: Molecular function.

\section{Declarations}

\section{Ethics approval}

Ethical approval or patient consent was unnecessary since the present study was an integrative analysis of published data.

\section{Consent for publication}


Not applicable

\section{Availability of data and materials}

The datasets used and/or analysed during the current study are available from dataset GSE111803 and GSE90728 in Gene Expression Omnibus (GEO).

\section{Competing interests}

The authors declare that they have no competing interests.

\section{Funding}

The present study was supported by Natural Science Foundation of Ningbo City (2019A610235).

\section{Authors' contributions}

XZ and KS performed the analysis of the data. LB and KS wrote the manuscript. XZ designed the study. All authors read and approved the final manuscript

\section{Code availability}

BioJupies (https://maayanlab.cloud/biojupies/)

miRPathDB 2.0( https://mpd.bioinf.uni-sb.de/)

TargetScan (http://www.targetscan.org/vert_71/)

DAVID (https://david.ncifcrf.gov/)

STRING (http://string.embl.de/)

Cytoscape (https://cytoscape.org/)

\section{Acknowledgements}

Not applicable

\section{References}

1. Siegel RL, Miller KD, Jemal A: Cancer statistics, 2019. CA: a cancer journal for clinicians 2019, 69(1):7-34.

2. Tomasetti M, Lee W, Santarelli L, Neuzil J: Exosome-derived microRNAs in cancer metabolism: possible implications in cancer diagnostics and therapy. Experimental \& molecular medicine 2017, 49(1):e285. 
3. Donnarumma E, Fiore D, Nappa M, Roscigno G, Adamo A, Iaboni M, Russo V, Affinito A, Puoti I, Quintavalle C et al: Cancer-associated fibroblasts release exosomal microRNAs that dictate an aggressive phenotype in breast cancer. Oncotarget 2017, 8(12):19592-19608.

4. Liu J, Wu S, Zheng X, Zheng P, Fu Y, Wu C, Lu B, Ju J, Jiang J: Immune suppressed tumor microenvironment by exosomes derived from gastric cancer cells via modulating immune functions. Scientific reports 2020, 10(1):14749.

5. Whiteside TL: Exosomes and tumor-mediated immune suppression. The Journal of clinical investigation 2016, 126(4):1216-1223.

6. Wang L, Chen X, Zhou X, Roizman B, Zhou GG: miRNAs Targeting ICP4 and Delivered to Susceptible Cells in Exosomes Block HSV-1 Replication in a Dose-Dependent Manner. Molecular therapy : the journal of the American Society of Gene Therapy 2018, 26(4):1032-1039.

7. Wang J, Ni J, Beretov J, Thompson J, Graham P, Li Y: Exosomal microRNAs as liquid biopsy biomarkers in prostate cancer. Critical reviews in oncology/hematology 2020, 145:102860.

8. Graner MW, Schnell S, Olin MR: Tumor-derived exosomes, microRNAs, and cancer immune suppression. Seminars in immunopathology 2018, 40(5):505-515.

9. Berchem G, Noman MZ, Bosseler M, Paggetti J, Baconnais S, Le Cam E, Nanbakhsh A, Moussay E, Mami-Chouaib F, Janji B et al: Hypoxic tumor-derived microvesicles negatively regulate NK cell function by a mechanism involving TGF- $\beta$ and miR23a transfer. Oncoimmunology 2016, 5(4):e1062968.

10. Walsh PT, Buckler JL, Zhang J, Gelman AE, Dalton NM, Taylor DK, Bensinger SJ, Hancock WW, Turka LA: PTEN inhibits IL-2 receptor-mediated expansion of CD4+ CD25+ Tregs. The Journal of clinical investigation 2006, 116(9):2521-2531.

11. Yao B, Qu S, Hu R, Gao W, Jin S, Liu M, Zhao Q: A panel of miRNAs derived from plasma extracellular vesicles as novel diagnostic biomarkers of lung adenocarcinoma. FEBS open bio 2019, 9(12):21492158.

12. Torre D, Lachmann A, Ma'ayan A: BioJupies: Automated Generation of Interactive Notebooks for RNA-Seq Data Analysis in the Cloud. Cell systems 2018, 7(5):556-561.e553.

13. Kehl T, Kern F, Backes C, Fehlmann T, Stöckel D, Meese E, Lenhof HP, Keller A: miRPathDB 2.0: a novel release of the miRNA Pathway Dictionary Database. Nucleic acids research 2020, 48(D1):D142-d147.

14. Agarwal V, Bell GW, Nam JW, Bartel DP: Predicting effective microRNA target sites in mammalian mRNAs. elife 2015, 4.

15. Huang da W, Sherman BT, Lempicki RA: Systematic and integrative analysis of large gene lists using DAVID bioinformatics resources. Nature protocols 2009, 4(1):44-57.

16. Szklarczyk D, Gable AL, Lyon D, Junge A, Wyder S, Huerta-Cepas J, Simonovic M, Doncheva NT, Morris JH, Bork P et al: STRING v11: protein-protein association networks with increased coverage, supporting functional discovery in genome-wide experimental datasets. Nucleic acids research 2019, 47(D1):D607-d613. 
17. Chin $\mathrm{CH}$, Chen $\mathrm{SH}, \mathrm{Wu} \mathrm{HH}, \mathrm{Ho} \mathrm{CW}$, Ko MT, Lin CY: cytoHubba: identifying hub objects and subnetworks from complex interactome. BMC systems biology 2014, 8 Suppl 4(Suppl 4):S11.

18. Hayes DA, Kunde DA, Taylor RL, Pyecroft SB, Sohal SS, Snow ET: ERBB3: A potential serum biomarker for early detection and therapeutic target for devil facial tumour 1 (DFT1). PloS one 2017, 12(6):e0177919.

19. Kuczek DE, Larsen AMH, Thorseth ML, Carretta M, Kalvisa A, Siersbæk MS, Simões AMC, Roslind A, Engelholm LH, Noessner $\mathrm{E}$ et al: Collagen density regulates the activity of tumor-infiltrating $\mathrm{T}$ cells. Journal for immunotherapy of cancer 2019, 7(1):68.

20. Xu D, Wang Y, Zhou K, Wu J, Zhang Z, Zhang J, Yu Z, Liu L, Liu X, Li B et al: Identification of an extracellular vesicle-related gene signature in the prediction of pancreatic cancer clinical prognosis. Bioscience reports 2020, 40(12).

21. Kimura K, Hohjoh H, Fukuoka M, Sato W, Oki S, Tomi C, Yamaguchi H, Kondo T, Takahashi R, Yamamura T: Circulating exosomes suppress the induction of regulatory $\mathrm{T}$ cells via let-7i in multiple sclerosis. Nature communications 2018, 9(1):17.

22. Knudson KM, Hicks KC, Luo X, Chen JQ, Schlom J, Gameiro SR: M7824, a novel bifunctional anti-PDL1/TGF $\beta$ Trap fusion protein, promotes anti-tumor efficacy as monotherapy and in combination with vaccine. Oncoimmunology 2018, 7(5):e1426519.

23. Bertrand F, Montfort A, Marcheteau E, Imbert C, Gilhodes J, Filleron T, Rochaix P, Andrieu-Abadie N, Levade T, Meyer $\mathrm{N}$ et al: TNFa blockade overcomes resistance to anti-PD-1 in experimental melanoma. Nature communications 2017, 8(1):2256.

24. Kerkar SP, Muranski P, Kaiser A, Boni A, Sanchez-Perez L, Yu Z, Palmer DC, Reger RN, Borman ZA, Zhang $L$ et al: Tumor-specific CD8+ T cells expressing interleukin-12 eradicate established cancers in lymphodepleted hosts. Cancer research 2010, 70(17):6725-6734.

25. Zhang Y, Wu L, Li Z, Zhang W, Luo F, Chu Y, Chen G: Glycocalyx-Mimicking Nanoparticles Improve Anti-PD-L1 Cancer Immunotherapy through Reversion of Tumor-Associated Macrophages. Biomacromolecules 2018, 19(6):2098-2108.

26. Yoon $\mathrm{H}$, Kim TS, Braciale TJ: The cell cycle time of $\mathrm{CD} 8+\mathrm{T}$ cells responding in vivo is controlled by the type of antigenic stimulus. PloS one 2010, 5(11):e15423.

27. O'Shea JJ, Holland SM, Staudt LM: JAKs and STATs in immunity, immunodeficiency, and cancer. The New England journal of medicine 2013, 368(2):161-170.

28. Ehsan M, Haseeb M, Hu R, Ali H, Memon MA, Yan R, Xu L, Song X, Zhu X, Li X: Tropomyosin: An Excretory/Secretory Protein from Haemonchus contortus Mediates the Immuno-Suppressive Potential of Goat Peripheral Blood Mononuclear Cells In Vitro. Vaccines 2020, 8(1).

29. Zou Y, Ruan S, Jin L, Chen Z, Han H, Zhang Y, Jian Z, Lin Y, Shi N, Jin H: CDK1, CCNB1, and CCNB2 are Prognostic Biomarkers and Correlated with Immune Infiltration in Hepatocellular Carcinoma. Medical science monitor : international medical journal of experimental and clinical research 2020, 26:e925289. 
30. Zhang YP, Bao ZW, Wu JB, Chen YH, Chen JR, Xie HY, Zhou L, Wu J, Zheng SS: Cancer-Testis Gene Expression in Hepatocellular Carcinoma: Identification of Prognostic Markers and Potential Targets for Immunotherapy. Technology in cancer research \& treatment 2020, 19:1533033820944274.

31. Zhang TY, Ren HY, Pan N, Dong HX, Zhao SM, Wen ZF, Wang XR, Wang LX: Tumor cell-derived autophagosomes (DRibbles)-activated B cells induce specific naïve CD8(+) T cell response and exhibit antitumor effect. Cancer immunology, immunotherapy : CII 2021, 70(2):463-474.

32. Ren H, Zhao S, Li W, Dong H, Zhou M, Cao M, Hu HM, Wang LX: Therapeutic antitumor efficacy of B cells loaded with tumor-derived autophagasomes vaccine (DRibbles). Journal of immunotherapy (Hagerstown, Md : 1997) 2014, 37(8):383-393.

33. Luo L, Lv M, Zhuang X, Zhang Q, Qiao T: Irradiation increases the immunogenicity of lung cancer cells and irradiation-based tumor cell vaccine elicits tumor-specific $T$ cell responses in vivo. OncoTargets and therapy 2019, 12:3805-3815.

34. Ivanoff A, Ivanoff J, Hultenby K, Sundqvist KG: Infiltrative capacity of T leukemia cell lines: a distinct functional property coupled to expression of matrix metalloproteinase-9 (MMP-9) and tissue inhibitor of metalloproteinases-1 (TIMP-1). Clinical \& experimental metastasis 1999, 17(8):695-711.

35. Vacchelli E, Ma Y, Baracco EE, Sistigu A, Enot DP, Pietrocola F, Yang H, Adjemian S, Chaba K, Semeraro $\mathrm{M}$ et al: Chemotherapy-induced antitumor immunity requires formyl peptide receptor 1 . Science (New York, NY) 2015, 350(6263):972-978.

36. Tian F, Wang J, Ouyang T, Lu N, Lu J, Shen Y, Bai Y, Xie X, Ge Q: MiR-486-5p Serves as a Good Biomarker in Nonsmall Cell Lung Cancer and Suppresses Cell Growth With the Involvement of a Target PIK3R1. Frontiers in genetics 2019, 10:688.

37. Tan X, Qin W, Zhang L, Hang J, Li B, Zhang C, Wan J, Zhou F, Shao K, Sun Y et al: A 5-microRNA signature for lung squamous cell carcinoma diagnosis and hsa-miR-31 for prognosis. Clinical cancer research : an official journal of the American Association for Cancer Research 2011, 17(21):68026811.

38. Yang S, Sui J, Liu T, Wu W, Xu S, Yin L, Pu Y, Zhang X, Zhang Y, Shen B et al: Expression of miR-486$5 p$ and its significance in lung squamous cell carcinoma. Journal of cellular biochemistry 2019, 120(8):13912-13923.

39. Yu YP, Landsittel D, Jing L, Nelson J, Ren B, Liu L, McDonald C, Thomas R, Dhir R, Finkelstein S et al: Gene expression alterations in prostate cancer predicting tumor aggression and preceding development of malignancy. Journal of clinical oncology : official journal of the American Society of Clinical Oncology 2004, 22(14):2790-2799.

40. Jin X, Chen Y, Chen H, Fei S, Chen D, Cai X, Liu L, Lin B, Su H, Zhao L et al: Evaluation of TumorDerived Exosomal miRNA as Potential Diagnostic Biomarkers for Early-Stage Non-Small Cell Lung Cancer Using Next-Generation Sequencing. Clinical cancer research : an official journal of the American Association for Cancer Research 2017, 23(17):5311-5319.

41. Sun B, Han Y, Shi M: Stromal-derived miR-486-5p promotes metastasis of non-small-cell lung cancer cells by targeting the CADM1/tight junctions axis in vascular endothelial cells. Cell biology 
international 2020.

42. Shindo Y, Hazama S, Nakamura Y, Inoue Y, Kanekiyo S, Suzuki N, Takenouchi H, Tsunedomi R, Nakajima M, Ueno T et al: miR-196b, miR-378a and miR-486 are predictive biomarkers for the efficacy of vaccine treatment in colorectal cancer. Oncology letters 2017, 14(2):1355-1362.

43. Zhou Z, Zhou X, Jiang Y, Qiu M, Liang X, Lin Q, Guo Q, Nong C, Huo R, Chen Q et al: Clinical significance of miR-1180-3p in hepatocellular carcinoma: a study based on bioinformatics analysis and RT-qPCR validation. Scientific reports 2020, 10(1):11573.

44. Chen M, Huang X, Li L, Huang M, Cai R, Liao X: A Regulatory Axis of circ_0008193/miR-11803p/TRIM62 Suppresses Proliferation, Migration, Invasion, and Warburg Effect in Lung Adenocarcinoma Cells Under Hypoxia. Medical science monitor : international medical journal of experimental and clinical research 2020, 26:e922900.

45. Zhu XL, Ren LF, Wang HP, Bai ZT, Zhang L, Meng WB, Zhu KX, Ding FH, Miao L, Yan J et al: Plasma microRNAs as potential new biomarkers for early detection of early gastric cancer. World journal of gastroenterology 2019, 25(13):1580-1591.

46. Willinger CM, Rong J, Tanriverdi K, Courchesne PL, Huan T, Wasserman GA, Lin H, Dupuis J, Joehanes R, Jones MR et al: MicroRNA Signature of Cigarette Smoking and Evidence for a Putative Causal Role of MicroRNAs in Smoking-Related Inflammation and Target Organ Damage. Circulation Cardiovascular genetics 2017, 10(5).

47. Liu J, Yoo J, Ho JY, Jung Y, Lee S, Hur SY, Choi YJ: Plasma-derived exosomal miR-4732-5p is a promising noninvasive diagnostic biomarker for epithelial ovarian cancer. Journal of ovarian research 2021, 14(1):59.

48. Zhang K, Zhao S, Wang Q, Yang HS, Zhu J, Ma R: Identification of microRNAs in Nipple Discharge as Potential Diagnostic Biomarkers for Breast Cancer. Annals of surgical oncology 2015, 22 Suppl 3:S536-544.

49. Zhang X, Bai J, Yin H, Long L, Zheng Z, Wang Q, Chen F, Yu X, Zhou Y: Exosomal miR-1255b-5p targets human telomerase reverse transcriptase in colorectal cancer cells to suppress epithelial-tomesenchymal transition. Molecular oncology 2020, 14(10):2589-2608.

50. Silva CA, Ribeiro-Dos-Santos A, Gonçalves WG, Pinto P, Pantoja RP, Vinasco-Sandoval T, Ribeiro-DosSantos AM, Hutz MH, Vidal AF, Araújo GS et al: Can miRNA Indicate Risk of Illness after Continuous Exposure to M. tuberculosis? International journal of molecular sciences 2021, 22(7).

51. Buchwald ZS, Yang C, Nellore S, Shashkova EV, Davis JL, Cline A, Ko J, Novack DV, DiPaolo R, Aurora R: A Bone Anabolic Effect of RANKL in a Murine Model of Osteoporosis Mediated Through FoxP3+ CD8 T Cells. Journal of bone and mineral research : the official journal of the American Society for Bone and Mineral Research 2015, 30(8):1508-1522.

52. Walsh MC, Kim N, Kadono Y, Rho J, Lee SY, Lorenzo J, Choi Y: Osteoimmunology: interplay between the immune system and bone metabolism. Annual review of immunology 2006, 24:33-63.

53. Nakashima T, Takayanagi $\mathrm{H}$ : The dynamic interplay between osteoclasts and the immune system. Archives of biochemistry and biophysics 2008, 473(2):166-171. 
54. Limmer A, Wirtz DC: Osteoimmunology: Influence of the Immune System on Bone Regeneration and Consumption. Zeitschrift fur Orthopadie und Unfallchirurgie 2017, 155(3):273-280.

55. Goldmann O, Medina E: Staphylococcus aureus strategies to evade the host acquired immune response. International journal of medical microbiology : IJMM 2018, 308(6):625-630.

56. Lee B, Olaniyi R, Kwiecinski JM, Wardenburg JB: Staphylococcus aureus toxin suppresses antigenspecific T cell responses. The Journal of clinical investigation 2020, 130(3):1122-1127.

57. Zhang Z, Yu Y, Zhang P, Ma G, Zhang M, Liang Y, Jiao W, Niu H: Identification of NTRK3 as a potential prognostic biomarker associated with tumor mutation burden and immune infiltration in bladder cancer. BMC cancer 2021, 21(1):458.

58. Yamauchi T, Hoki T, Oba T, Saito H, Attwood K, Sabel MS, Chang AE, Odunsi K, Ito F: CX3CR1-CD8+ T cells are critical in antitumor efficacy but functionally suppressed in the tumor microenvironment. $\mathrm{JCl}$ insight 2020, 5(8).

59. Kee JY, Ito A, Hojo S, Hashimoto I, Igarashi Y, Tsukada K, Irimura T, Shibahara N, Nakayama T, Yoshie 0 et al: Chemokine CXCL16 suppresses liver metastasis of colorectal cancer via augmentation of tumor-infiltrating natural killer T cells in a murine model. Oncology reports 2013, 29(3):975-982.

60. Matsumura S, Wang B, Kawashima N, Braunstein S, Badura M, Cameron TO, Babb JS, Schneider RJ, Formenti SC, Dustin ML et al: Radiation-induced CXCL16 release by breast cancer cells attracts effector T cells. Journal of immunology (Baltimore, Md : 1950) 2008, 181(5):3099-3107.

61. Fan T, Zhu M, Wang L, Liu Y, Tian H, Zheng Y, Tan F, Sun N, Li C, He J: Immune profile of the tumor microenvironment and the identification of a four-gene signature for lung adenocarcinoma. Aging 2020, 13(2):2397-2417.

62. Lin J, Wu C, Ma D, Hu Q: Identification of P2RY13 as an immune-related prognostic biomarker in lung adenocarcinoma: A public database-based retrospective study. PeerJ 2021, 9:e11319.

63. Dai D, Xie L, Shui Y, Li J, Wei Q: Identification of Tumor Microenvironment-Related Prognostic Genes in Sarcoma. Frontiers in genetics 2021, 12:620705.

64. Sung JY, Cheong JH: Pan-Cancer Analysis Reveals Distinct Metabolic Reprogramming in Different Epithelial-Mesenchymal Transition Activity States. Cancers 2021, 13(8).

65. Di Virgilio F, Sarti AC, Falzoni S, De Marchi E, Adinolfi E: Extracellular ATP and P2 purinergic signalling in the tumour microenvironment. Nature reviews Cancer 2018, 18(10):601-618.

66. Liu B, Wang Z, Gu M, Zhao C, Ma T, Wang J: GEO Data Mining Identifies OLR1 as a Potential Biomarker in NSCLC Immunotherapy. Frontiers in oncology 2021, 11:629333.

67. Onishi H, Kiyota A, Koya N, Tanaka H, Umebayashi M, Katano M, Morisaki T: Random migration contributes to cytotoxicity of activated CD8+ T-lymphocytes but not NK cells. Anticancer research 2014, 34(8):3947-3956.

68. Priceman SJ, Shen S, Wang L, Deng J, Yue C, Kujawski M, Yu H: S1PR1 is crucial for accumulation of regulatory T cells in tumors via STAT3. Cell reports 2014, 6(6):992-999. 
69. Hope C, Emmerich PB, Papadas A, Pagenkopf A, Matkowskyj KA, Van De Hey DR, Payne SN, Clipson L, Callander NS, Hematti $P$ et al: Versican-Derived Matrikines Regulate Batf3-Dendritic Cell Differentiation and Promote T Cell Infiltration in Colorectal Cancer. Journal of immunology (Baltimore, Md : 1950) 2017, 199(5):1933-1941.

70. Ajona D, Ortiz-Espinosa S, Moreno H, Lozano T, Pajares MJ, Agorreta J, Bértolo C, Lasarte JJ, Vicent $\mathrm{S}$, Hoehlig $\mathrm{K}$ et al: A Combined PD-1/C5a Blockade Synergistically Protects against Lung Cancer Growth and Metastasis. Cancer discovery 2017, 7(7):694-703.

71. Gibson JT, Orlandella RM, Turbitt WJ, Behring M, Manne U, Sorge RE, Norian LA: Obesity-Associated Myeloid-Derived Suppressor Cells Promote Apoptosis of Tumor-Infiltrating CD8 T Cells and Immunotherapy Resistance in Breast Cancer. Frontiers in immunology 2020, 11:590794.

\section{Figures}

A

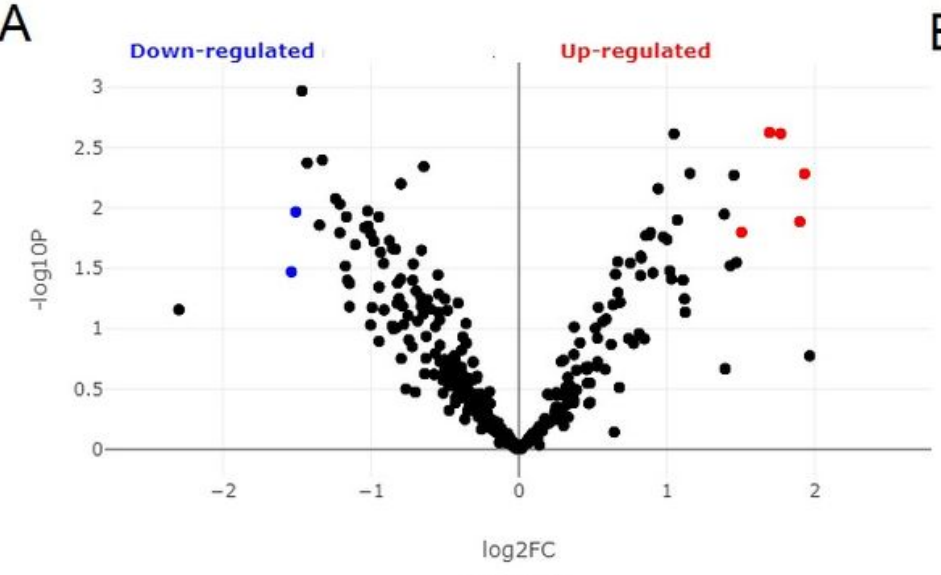

DEMs
B

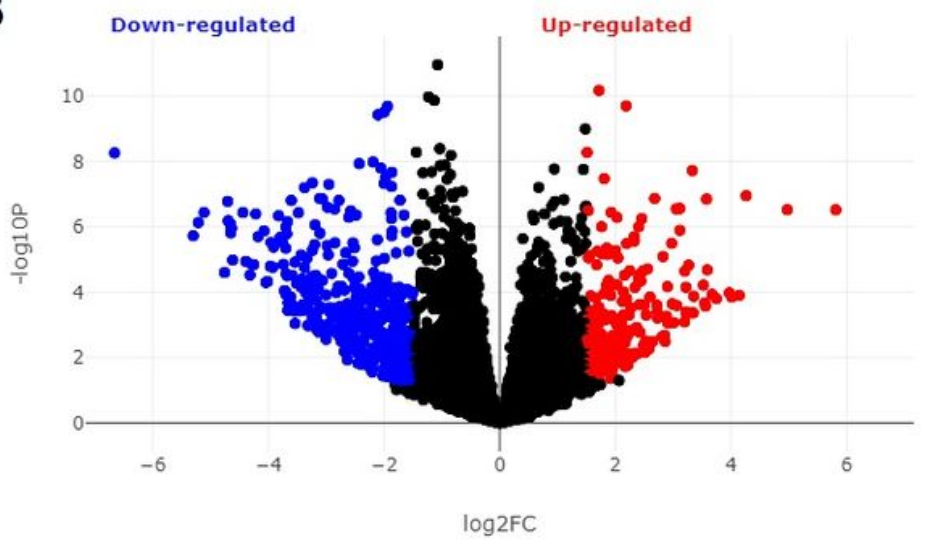

DEGs

Figure 1

The volcano plot of DEMs and DEGs

(A) The volcano plot of DEMs,(B) The volcano plot of DEGs 


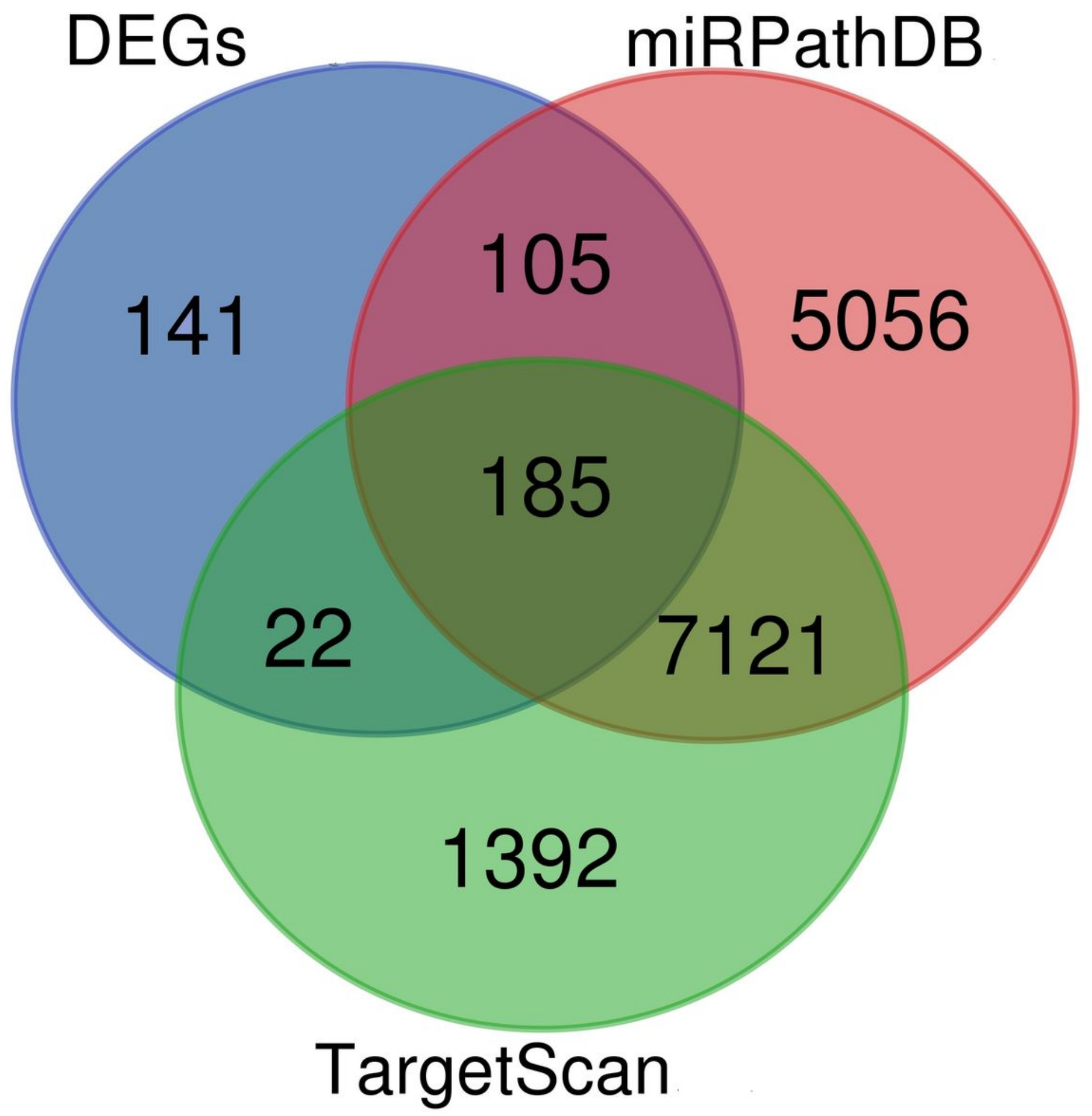

Figure 2

Venn plot for DEGs and target genes of DEMs 


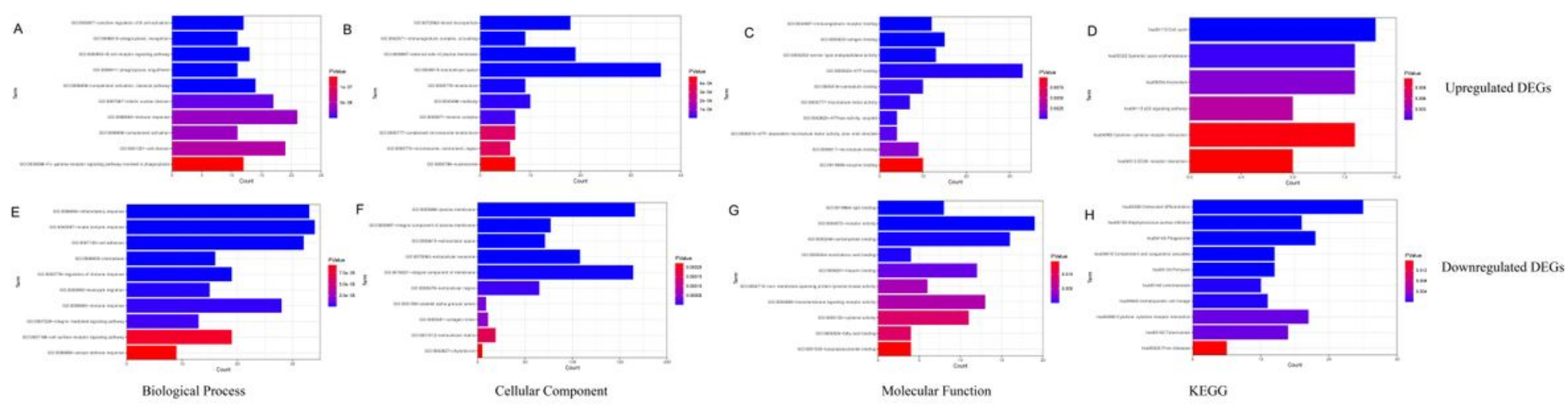

Figure 3

GO and KEGG analysis for DEGs.

(A) BP group for upregulated DEGs,

(B) CC group for upregulated DEGs,

(C) MF group for upregulated DEGs,

(D)KEGG for upregulated DEGs,

(E) BP group downregulated DEGs,

(F) CC group for downregulated DEGs,

(G) MF group for downregulated DEGs,

(H) KEGG for downregulated DEGs
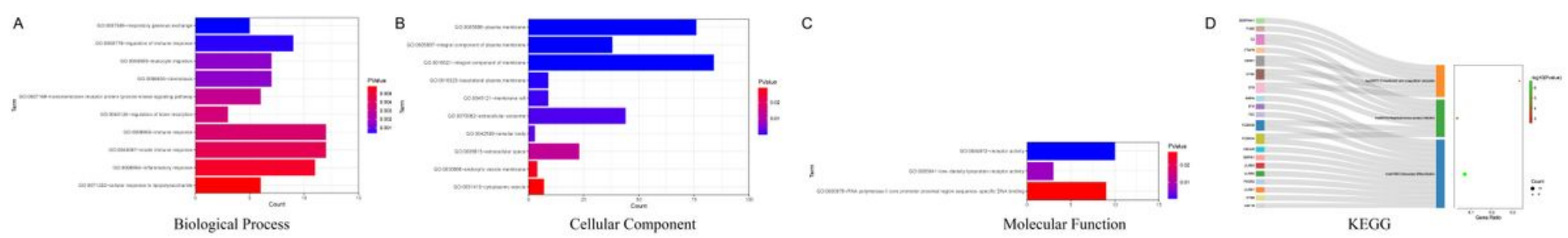

Figure 4

GO and KEGG analysis for overlapped target genes.

(A) BP group for overlapped target genes, (B) CC group for overlapped target genes, (C) MF group for overlapped target genes, (D) KEGG for overlapped target genes. 
A

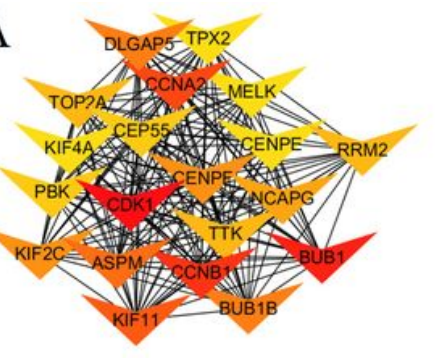

D
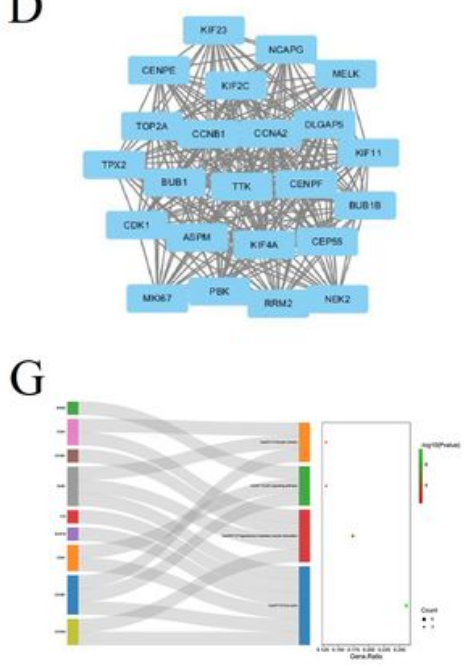

Upregulated DEGs
B

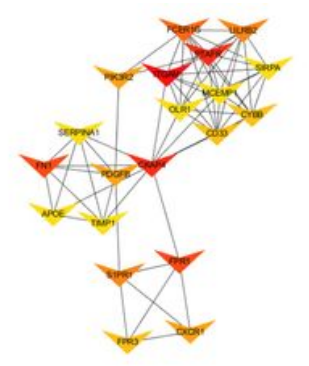

E

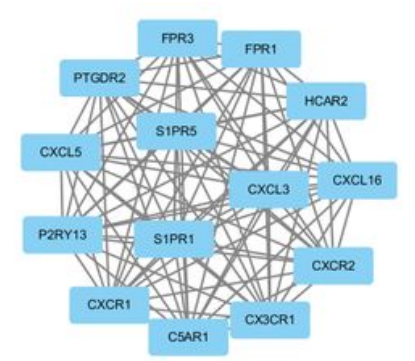

$\mathrm{H}$

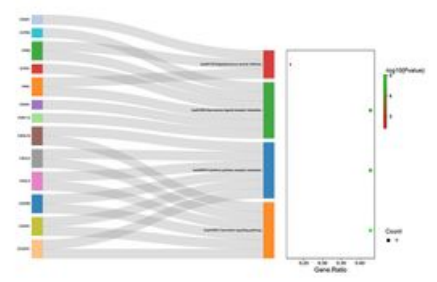

Downregulated DEGs
C

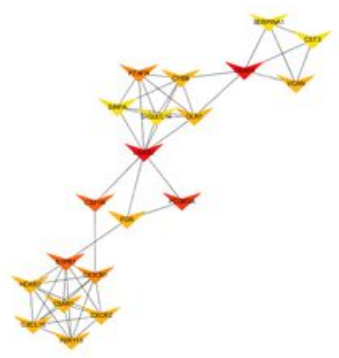

F

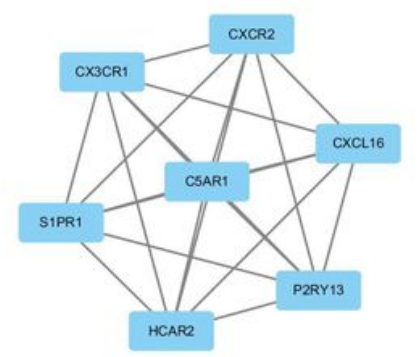

I

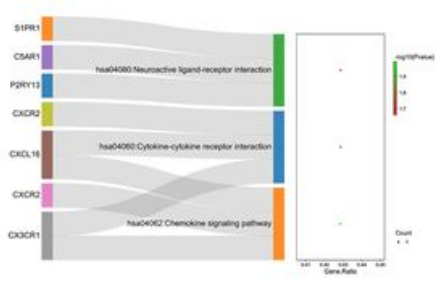

Overlapped target genes
Hub genes

Modules

KEGG pathways

\section{Figure 5}

Hub genes and modules analysis

(A) The top 20 ranked hub genes for upregulated DEGs set, (B) The top 20 ranked hub genes for downregulated DEGs set, (C) The top 20 ranked hub genes for overlapped target genes, (D) A significant module for upregulated DEGs, (E) A significant module for downregulated DEGs, (F) A significant module for overlapped target genes. (G) KEGG pathway on the module for upregulated DEGs, (H) KEGG pathway on the module for downregulated DEGs (I)KEGG pathway on the module for overlapped target genes. 


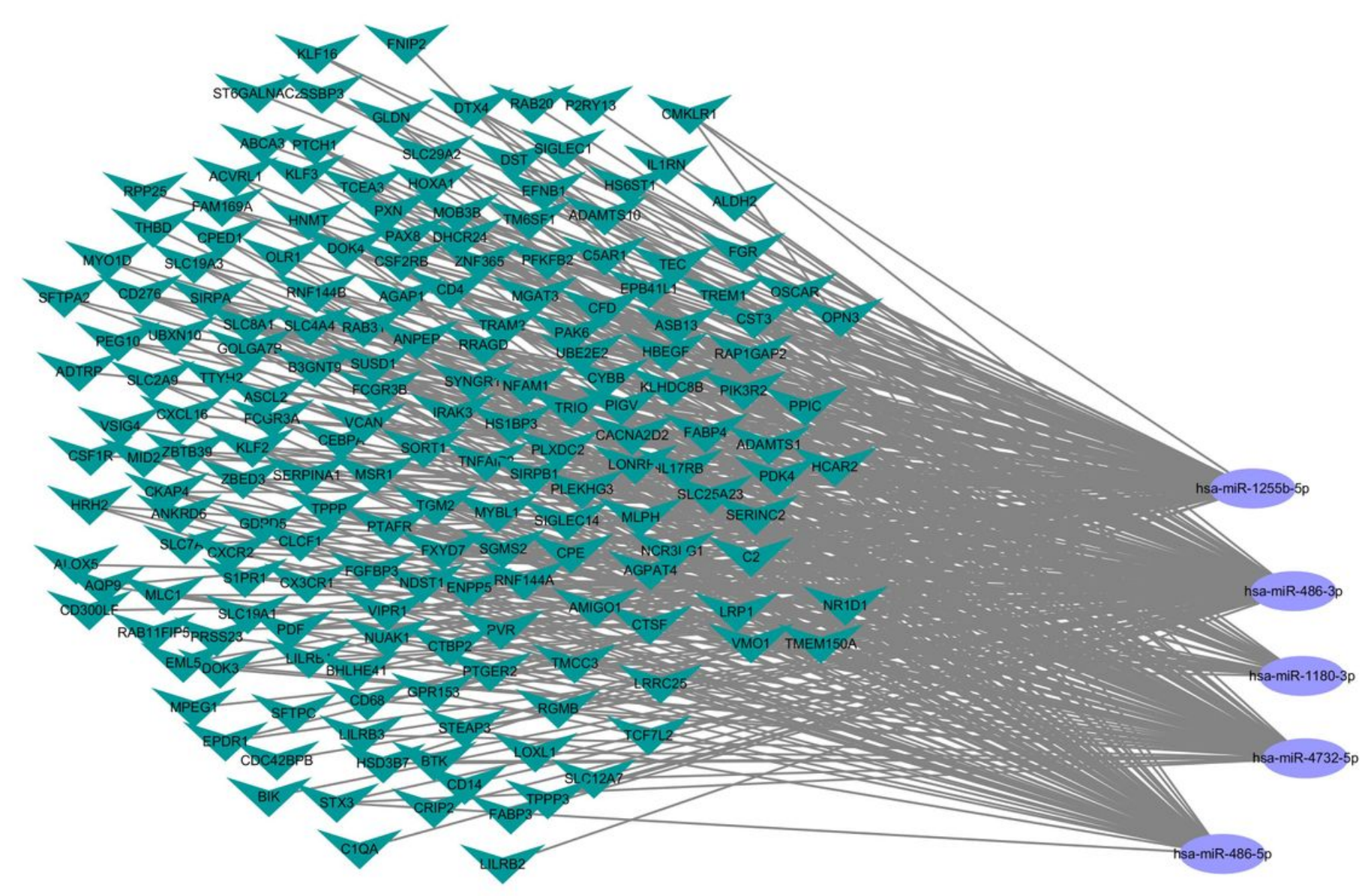

Figure 6

Regulatory network of upregulated DEMs and overlapped target genes,

The ellipse nodes indicate upregulated DEMs. V-shape nodes represent overlapped target genes DEMs.

\section{Supplementary Files}

This is a list of supplementary files associated with this preprint. Click to download.

- SupplementaryMaterial1.txt 